hexagonale Pyramide der Diagonale

Galenoïd stumpfe Rhomboëder in verkehrter Stellung spitze Rhomboëder in verkehrter Stellung $\quad$ om $=\mathrm{m} 1 \mathrm{~m}$ verkehrtes Grundrhomboëder $02=\overline{212}$ Skalenoëder der Diagonale $\overline{0} \mathrm{~m}=\overline{\mathrm{m}} \mathrm{m} 1$ hexagonale Pyramide der Diagonale

Adamantoïd stumpfe Skalenoëder

$\overline{0} 3=\overline{331}$ spitze Skalenoëder $\quad 0 s= \pm m \pm n 1$
stumpfe hexagon. Pyramiden os ${ }^{1}=m \frac{m \pm 1}{2} 1$ os $=\mathrm{mn} 1$ spitze hexagon. Pyramiden os ${ }^{1}=\bar{m} \frac{m+1}{2} 1$ zwölfseitiges Prisma $\quad \underline{0} \mathrm{~s}^{11}=\overline{\mathrm{m}} \cdot \mathrm{m}-1 \cdot 1$.

(Prager Sitzungsber. 1872. 1. 61-87. Zeitschr. f. d. gesammt. Naturwissensch. von Giebel. Neue Folge 1873. Bd. VII. pag. 304.).

C. Sch.

\title{
Vorkommen von Bleisulfureten und Braunkohlen in Griechenland.
}

Das an Blei reichste Land ist nach Landerer unstreitig wohl Griechenland, obgleich jährlich für Millionen Frs. Bleiweiss eingeführt wird, könnte es doch mit seinem Reichthum an reinen und silberhaltigen Bleisulfureten ganz Europa versorgen und für Millionen davon ausfuihren. An mehr als 30 Plätzen, so wie auf den meisten Inseln des griechischen Archipels, im Peleponese, in Akarnanien und auch auf den jonischen Inseln werden viele Meilen weit ausgedehnte Einlagerungen von silberhaltigen Bleisulfureten aufgefunden, die sich bis unter den Meeresspiegel erstrecken. Hier und da sind ganze Lager von Bleisulfureten mit Weissbleierz ïberzogen, 'welche beim Ausschmelzen im Grossen 40-50\% silberhaltiges Blei liefern.

Auf den Inseln Seriphos, Keos, Samos und Antiparos, finden sich solche Lager von Bleisulfureten gemengt mit Zinksulfureten und Galmei. 


\section{Die Brannkohlen Griechenlands}

sind nicht mit den englischen zu vergleichen. Sie scheinen ein Alter von 6-8000 Jahren zu haben und stammen von Pinien - Abies - Platanus - Acer - Oliven - Quercus Arbutus, Andrachne und Populus-Arten ab, welches auch noch die Hauptbüume der heutigen Flora sind. Die Kohlen eignen sich zu allen häuslichen und industriellen Zwecken.

$$
\text { C. Sch. }
$$

\section{Erze Griechenlands.}

Ueber das Vorkommen von Eisenerzen theilt Landerer mit, dass sich solche in Griechenland in grosser Menge vorfinden und dass vorziglich Brauneisenstein und Eisenglanz in solcher Menge vorhanden, dass ganz Europa für lange Zeit seinen Eisenbedarf daraus gewinnen könne. Zum Zwecke der Gewinnung des Metalls hat sich eine Gesellschaft gebildet, jedoch ist bis jetzt wegen mangelnden Steinkohlen noch kein gutes Resultat erhalten. Nach England ausgeführte und dort verarbeitete Erze lieferten 60-65\% gutes Eisen. Es kommt nun darauf an, was vortheilhafter ist, die griechischen Eisenerze auszuführen, oder Steinkohlen und Coakes nach Griechenland einzufuihren.

Ebenfalls erfreut sich Griechenland eines grossen Reichthums an silberhaltigen Bleierzen, die sich nach Herrn Landerer hauptsächlich im Pelopones, sowie auf den griechischen Inseln finden. In Griechenland selbst wird nur das Blei ausgeschmolzen und nach England und Frankreich, in welchen beiden Ländern dasselbe auf seinen Silbergehalt verarbeitet wird, ausgeführt. Es sollen 100 Pfund Blei ca. 30,0 g. Silber enthalten, was einem Procentgehalt von 0,06 entspricht.

Auf den Inseln Chios und Samos sind in neuester Zeit Antimonerze anfgefunden, auf Samos zugleich auch Zinkerze. Zur Ausbeutung dieser Vorkommnisse hat sich eine Gesellschaft gebildet, der es jedoch sehr an tiuchtigen Bergbauverständigen mangelt. Man sucht desshalb fremde Kräfte heranzuziehen und hat hierbei sein Hauptaugenmerk auf deutsche Bergbaubeamte gerichtet, denen man sehr hohe Gehälter, von 4 bis 600 Thlr. per Monat bietet.

Ausser den schon angefuihrten Vorkommnissen schätzbarer Mineralien führt Landerer noch den sogenannten 